\title{
Technology for Robotic Surface Inspection in Space
}

\author{
Richard Volpe* \\ J. Balaram ${ }^{\dagger}$ \\ Jet Propulsion Laboratory \\ California Institute of Technology \\ Pasadena, California 91109
}

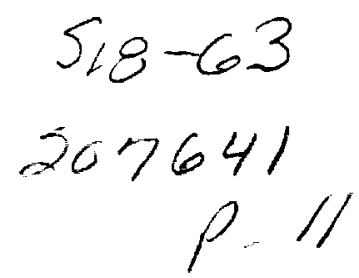

\begin{abstract}
This paper presents on-going research in robotic inspection of space platforms. Three main areas of investigation are discussed: machine-vision inspection techniques, an integrated sensor end-effector, and an orbital environment laboratory simulation. Machine-vision inspection utilizes automatic comparison of new and reference images to detect on-orbit induced damage such as micro-meteorite impacts. The cameras and lighting used for this inspection are housed in a multisensor end-effector, which also contains a suite of sensors for detection of temperature, gas leaks, proximity, and forces. To fully test all of these sensors, a realistic space platform mock-up has been created, complete with visual, temperature, and gas anomalies. Further, changing orbital lighting conditions are effectively mimicked by a robotic solar simulator. In the paper, each of these technology components will be discussed, and experimental results are provided.
\end{abstract}

\section{Introduction}

Later this decade, NASA will place in orbit around Earth the Space Station Freedom (SSF), which will be used as a science station and home for astronauts for 30 years. Soon after its initial design, engineering reviews revealed that simple inspection and maintenance of the station would consume more time than the astronauts would have available [2]. This was reinforced by results of the Long Duration Exposure Facility (LDEF), which showed large amounts of damage from micro-meteorite impacts and atomic oxygen degradation while in orbit for five years [8]. For these reasons, NASA sponsored The Remote Surface Inspection Task (RSI), a five year technology demonstration task at the Jet Propulsion Laboratory, California Institute of Technology (JPL). This project has developed and systematically investigated methods for telerobotic inspection of SSF [4].

*email: volpe@telerobotics.jpl.nasa.gov

†email: balaram@telerobotics.jpl.nasa.gov
The inspection system which has been built for this research is comprised of three main subsystems: robot manipulator control, graphical user interfacing, and teleoperated/automated multi-sensor inspection. The robot manipulator subsystem is comprised of a Robotics Research K1207 arm mounted on a translating platform, and controlled by a real-time system employing Configuration Control [9]. The graphical user interface subsystem resides on an SGI workstation and provides user-friendly interfaces to the manipulator control and the inspection data [6]. The multi-sensor inspection subsystem analyzes a realistic SSF mockup under simulated orbital conditions, gathering sensory data indicative of potential problems. This inspection subsystem is the topic of this paper. The key technology items addressed are: methods for automated visual inspection; the development of an Integrated Sensor End-Effector (ISEE) which encompasses vision, proximity, temperature, and gas information to monitor the environment; and a high fidelity simulation of orbital inspection conditions. In this paper, each of these will be described as well as the issues which they successfully address.

The paper is organized as follows: Section 2 discusses automated visual inspection in detail, including the issues of ambient light and registration error compensation, as well as flaw and error models. Section 3 describes the ISEE, and provides a detailed discussion of the use of proximity sensors for collision avoidance and surface following. Section 4 discusses the simulated conditions for the inspection operations, including a description of the SSF truss mock-up and its temperature and gas anomalies, as well as a solar simulator which provides realistic orbital lighting conditions. Finally, Section 5 provides a summary and some conclusions drawn from this technology development research.

\section{Visual Inspection}

The approach adopted for on-orbit inspection of space platforms consists of locating and characterizing flawinduced changes between an earlier reference image 
and a new inspection image. In the absence of noise, viewpoint differences, lighting variations, and benign changes, the detection of significant new damage could be obtained by a process of simple differencing. However, on-orbit use of robotic machine-vision to achieve this goal is constrained by a number of technical challenges:

- Imaging Repeatability. Subsequent scans of the space platform will not be able to achieve the same imaging view-point because of the lack of robot positioning repeatability and the expansion/contraction of space platform structures. This can result in mis-registered reference and inspection data sets, as well as previously occluded features being made visible and mistaken for new flaws. The presence of the flaw itself can complicate the recognition of the extent of the misregistration.

- Lighting Variation. In orbit, surface appearance can change drastically due to the variation in ambient light (solar and earthlight) illumination induced by orbital motion. Power constraints on artificial illuminators restrict the illumination techniques that can be adopted to compensate for this variability. Furthermore, the lack of atmospheric dispersion of the harsh solar light results in images having a large dynamic range with sharp shadows.

- Flaw and Object Appearance. The surface flaws caused by micro-meteorite damage are very small $(\approx 1 \mathrm{~mm})[7]$ and must be detected on man-made objects with complex geometric shapes and constructed with specular materials. Benign changes such as the gradual reflectivity variation resulting from exposure to ultra-violet radiation and atomic-oxygen can mislead the inspection system.

- System Constraints. Efficient computer processing is a must, given the computational limitations imposed by the need to use compact, light-weight, low-power, space-qualified computers. Communication limitations in sending data back to Earth must also be considered in deciding on the partitioning of the image processing functions between the spacecraft and the ground. Data storage of the various reference images is less of a problem than would initially appear, thanks to the availability of space-qualified mass storage devices.

- Motion Constraints. Robot motions can induce significant platform disturbances due to robot start/stop motions. If the disturbance is to be minimized by performing all of the imaging from a continuously moving sensor platform, then the resulting problems of motion blur must be addressed.

In this report the focus is mainly on the effects of ambient light variability and image mis-registration, and the methods used to compensate for them. A brief discussion on flaw-models and the quantification of the flaw detection performance is also presented. A detailed presentation may be found in reference [1].

\subsection{Laboratory Imaging System}

The imager consists of an industrial color Charge Coupled Device (CCD) camera. With solar illumination at earth orbit at approximately 130000 lux, the total illumination on a typical inspection scene area of $0.1 \mathrm{~m}^{2}$ over the duration of a single video field $(1 / 60 \mathrm{~s})$ is approximately 215 lumen $\cdot \mathrm{s}$. This is many times that which can be provided by a low-powered artificial light source, especially if it were a continuous illuminator. Instead, artificial illumination is provided by an electronic strobe unit, with the laboratory unit providing an illumination of 1.3 lumen $\cdot \mathrm{s}$. When the strobe is used with the electronic shutter in the camera set to $1 / 10000 \mathrm{~s}$, the total ambient solar illumination of the scene is only 1.5 lumen $\cdot \mathrm{s}$, making it comparable to the strobe provided illumination. Note that the total strobe illumination remains unaffected by the electronic shutter activation because the strobe duration $(\approx 20 \mu \mathrm{s})$ is still much shorter than the exposure duration. The overall energy consumption for strobe lighting is also lower since the strobe is only used when the sensing platform traverses a new view-point. Further, the use of a short exposure time reduces the effects of motion-blur in degrading the images. (As a practical note, since the laboratory ambient light simulator, described in Section 4, cannot achieve full solar intensity, the camera electronic shutter is operated at a somewhat larger setting. This effectively achieves the same ambient-to-artificial illumination ratio relevant to orbital operations.)

The camera is operated with a unity gamma response. Any deviations from a linear response are compensated for in the digitizer. Linear response ensures that image intensity is proportional to scene radiance and allows linear operations (e.g. subtraction) on image fields to be correctly computed. This is required for the ambient light variability compensation methods discussed in the next section. All imaging is performed using only the luminance signal of the video signal (quantized to 8 bits) with the color subcarrier information suppressed. 


\subsection{Ambient Light Compensation}

Ambient light subtraction uses two image data sets to obtain a compensated image. The first data set is illuminated only with the ambient light and the second is illuminated with the ambient light as well as the artificial illuminator. The information in the first data set is subtracted from that in the second to give a compensated image that appears as if it were taken with the artificial illuminator alone. In order for the subtraction results to be valid, the sensor response is required to be linear. There is, however, a reduction of the signalto-noise $(\mathrm{S} / \mathrm{N})$ ratio since the subtraction process can nearly double the noise power in the data. Further, the utilization of the dynamic range of the camera is also reduced since the sensor cannot be allowed to saturate when both ambient and artificial illumination is utilized. The performance of ambient light subtraction is enhanced when the artificial illuminator provides energy comparable to (or more than) the ambient light. As discussed earlier, the electronic shuttering mechanism achieves this. Note that strobe illumination is essential here for operating with a moving imaging platform since continuous illuminators, even if low power and high-intensity, would take a finite amount of time to ramp up to the desired intensity level. This would require the imaging platform to be stationary during the taking of the two image data sets necessary to achieve compensation.

An additional problem is that in a strobe illuminated image only one of the $2: 1$ interleaved image fields (say the odd-field) is lit by the strobe, while both fields are ambient lit. An estimate of the ambient light component in the odd-field is generated from an average of the ambient light data in the even-field immediately above and below each odd-field image scan line. A compensated image is generated by intra-frame subtraction, wherein this ambient light estimate is subtracted from the odd-field data.

This process does suffer from some disadvantages, namely a halving of the vertical resolution in the compensated image and the possibility of interpolation errors when estimating the ambient-lit component of the image. As expected, if the same ambient light is used in the reference and inspection images, then the interpolation errors are identical and cancel when performing the subsequent image comparisons for flaw detection. Any non-zero change can then be attributed to the presence of a new flaw.

However, interpolation errors are of consequence when the ambient light changes, and lead to an increased probability of false errors during the flaw detection process. For two special cases which correspond to limiting cases typically encountered in real applica- tions, the deleterious effects of the interpolation error is manageable. The first case corresponds to when the ambient light illumination of the surface for both the reference and inspection images has low spatial variation and the underlying reflectivity of the surface undergoes a large change. Here analysis shows that the significant errors only happen in regions where the reflectivity changes are large, which are precisely the same regions where mis-registration errors due to sensor-toplatform positioning errors can be expected to be of greater significance.

The second case occurs with ambient light discontinuities at shadow boundaries. If the transition from light to dark in the "pen-umbra" region of the shadow is very sharp, then the estimate generated by interpolating the even-field data will be incorrect. If, however, the transition occurs over a spatial extent of more than a couple of pixels, then the interpolation process will be able to accurately estimate the ambient light in the middle of the shadow boundary region. The extent of the pen-umbra region is a function of the distance from the surface to the object casting the shadow. If the object is close to the surface then the transition is sharp, and conversely if it is far away from the surface the transition is more smooth. Assume that a pen-umbra region greater than 2 pixels is of sufficient spatial extent to permit the interpolation to be reasonably accurate. An estimate of the corresponding object distance that would generate such a shadow can be easily obtained from simple geometrical arguments. For a typical fieldof-view and imaging standoff-distance, a shadow transition region of 2 pixels corresponds to to a surface spatial extent of about $1 \mathrm{~mm}$. Noting that the sun subtends approximately 0.01 radians, and that the shadow penumbra must necessarily subtend the same angle, gives the corresponding object distance as being $0.1 \mathrm{~m}$. Thus sharp shadows will only be cast by objects closer than $0.1 \mathrm{~m}$ to the surface. Even for such sharp shadows, the situation is ameliorated by the fact that the resulting interpolation errors are localized to a region along the shadow boundary that has a very narrow width. If the flaw being detected has a spatial extent larger than this width, then the resulting errors during flaw detection are reduced. This issue is discussed further in the Section on flaw models (Section 2.4).

\subsection{Registration Error}

Registration errors are induced by the lack of repeatability in the viewpoints at which images are taken for the reference and inspection images. These viewpoint discrepancies arise due to the inherent accuracy limitations of moving camera platforms. In the laboratory 
environment, i.e., fixed targets and industrial arms with good repeatability, the inaccuracy translates to no more that one to two pixels when images are taken from relatively short distances of less than $0.7 \mathrm{~m}$. In the space environment, larger repeatability problems are to be expected due to arm flexibility and object location changes due to thermal expansion and structural flexibility.

With this mis-registration, the comparison of compensated images by performing a simple subtraction of the compensated reference and inspection images results in a number of "false edges" in the differenced image. The magnitude of registration error depends on both the directional gradient of the gray-level image with respect to the camera platform motion parameters, as well as the occlusions at each imaged point. Here, only the directional gradient with respect to lateral and horizontal motion of the camera platform are considered, since these are expected to dominate for this inspection application. Occlusion induced errors are also not considered, even though their effects could be significant near any sharp depth deviations in the image.

A Gauss-Newton iterative method is used to perform reference-to-inspection image registration prior to making the comparison. The residual sum of squares between the actual and an estimated picture is used as an evaluation function to indicate the degree of match between the inspection data and a transformed reference image. The objective is to find a suitable transformation of the reference image so that the residual is close to zero. The Gauss-Newton algorithm solves this nonlinear least-squares problem via an iterative solution method and exploits the special structure of the gradient and Hessian matrix of the evaluation function [3]. The iteration process is continued until the leastsquares residue drops below an acceptable threshold, at which point the estimate can be considered to be registered with the data. Note that terms involving the Jacobian matrix in the case of pure translational mis-registrations can be pre-computed resulting in significant run-time computational savings. Nevertheless, residual mis-registration is still possible because of early termination of iterative registration correction necessitated by real-time deadline processing constraints.

\subsection{Flaw and Error Models}

The process used to detect a flaw is intimately linked to the corresponding model of the flaw. Flaw models must provide a reasonable approximation to the physical appearance of the flaw while not being overly complex to preclude implementation of the associated flaw detec- tion algorithms on a real-time system. Two types of flaw models are presented and the corresponding flaw detection processes are characterized.

A single-pixel flaw model treats each individual pixel independently of other pixels when it comes to flaw determination. A flaw is assumed to be present at a pixel if the surface intensity at that pixel in the inspection image differs from the surface intensity in the corresponding reference image pixel by a value greater than a characteristic flaw strength. The characteristic flaw strength is a function of the flaw type and can be determined by examining images of known and/or calibrated flaws.

In a multi-pixel flaw model a flaw is assumed to be present at a pixel if it occupies a certain minimal spatial extent. More precisely, consider for both the inspection and reference images, the corresponding surface intensity vectors each comprised of the intensity values in a spatially connected region around that pixel. A flaw is assumed to be present at the pixel if these vectors differ from each other by greater than a flaw strength vector. Once again, characteristic flaw strength vectors are a function of the flaw type and can be determined by examining images of known and/or calibrated flaws.

Two special cases may be considered depending on the nature of the flaw model vector. The first of these, is the uniform flaw model which takes each component of the flaw strength vector to be equal. This model is suitable in cases where the flaw has a uniform appearance across the entire neighborhood (e.g. a spot of paint on a surface). The second is the peak/adjacent flaw model which takes all but one component of the flaw vector to be constant with the exception being one single component which has a higher absolute magnitude value than the others. The second type is suitable where the flaw has a strong peak value surrounded by adjacent pixels with smaller but uniform values. This provides a crude approximation to the flaw morphology of micro-meteoroid impact craters where the center of the crater is darker than the rest.

Given the definition of a flaw, the null decision hypothesis $\mathcal{H}_{0}$ assumes that there is no flaw. The flaw decision hypothesis $\mathcal{H}_{1}$ assumes that a flaw is superposed onto the reference image. In order to determine if a flaw is present, the log likelihood ratio [10] is checked to see if it exceeds the test threshold.

Working out the details in the single-pixel case indicates that, as expected, given compensated images corresponding to reference and inspection images, the flaw detection can be performed by locating flaws at all pixel locations where the differenced image exceeds a predetermined threshold. For the multi-pixel model, the flaw detection process involves taking weighted sums of 
the differenced image in a suitable window and comparing these sums to a pre-determined threshold. A sub-optimal version of the detection test can be implemented using morphological erosion operations.

With the appropriate model for the flaw, the theoretical flaw detection performance can be analyzed. The performance is dependent on the distribution of the flaw detection signal under the two competing hypothesis: the Null Hypothesis $\mathcal{H}_{0}$ and the Flaw Hypothesis $\mathcal{H}_{1}$. If these distributions do not overlap, then it is possible to pick a threshold parameter for the detection process such that all flaws that occur are detected, and at the same time no false-alarms are generated. However, the distributions of the signal under both hypothesis do overlap because of the nature of the noise in the imaging process, and as a consequence for any threshold parameter, there will always be a possibility of missing a flaw and of falsely identifying a flaw. The selection of the threshold affects the performance of the system and is a function of the characteristic flaw strength and the noise levels in the system. Too high a threshold will decrease the probability of detection $P_{D}$, while too low a threshold will increase the probability of a false alarm $P_{F}$. This aspect of the performance is captured by providing parametric plots of the $P_{D}$ versus $P_{F}$ for various cases. These plots are known as Receiver $O p$ erator Characteristics (ROC's) from their earlier use in radar target detection. A detail analysis of performance has been conducted using these concepts and presented elsewhere [1].

Errors in mis-registration correction and ambient light compensation can be interpreted as increasing the noise in the image leading to lower detection performance. Residual mis-registration errors induce a change in intensity which can be confused with a flaw. Only translational mis-registration effects are considered here since any mis-registration effects arising due to small angular motion in the image plane may be locally approximated as a translational mis-registration. An analysis shows that the intensity difference at a pixel due to mis-registration may be considered as an additional noise term that adds on to the more typical random noise components present in an image. The presence of mis-registration increases the threshold which must be exceeded before a difference value is considered to be a flaw, and consequently reduces the possible performance. In a similar way, interpolation errors during ambient light compensation can also be interpreted as a noise term distributed over the image. If these noise effects are localized then they have less of an impact on the multi-pixel flaw model likelihood-ratio test than on the single-pixel case. This is because of the averaging inherent in determining the likelihood ratio test in the

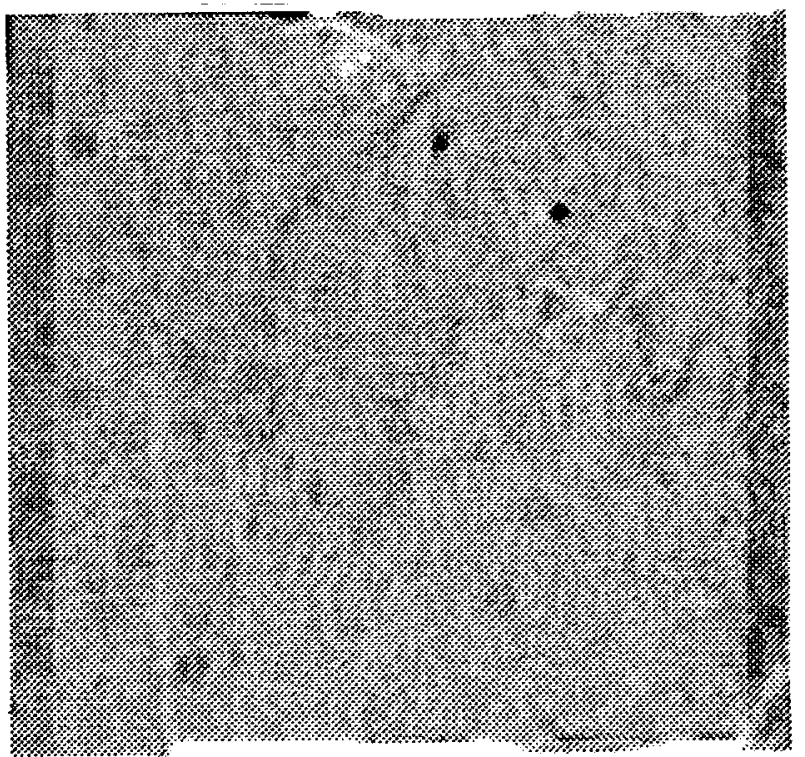

Figure 1: Large residuals are detected at flaw locations.

multi-pixel case.

A number of tests under different lighting conditions have been performed to test the flaw detection algorithms. Flaws are simulated by a random dot pattern of a given pixel size distributed on the surface of a test object. Figure 1 shows the final differenced image after mis-registration correction.

\subsection{Visual Inspection Summary}

The key conclusions are summarized:

- Image differencing appears to be a viable approach for flaw detection with the use of ambient light compensation methods and iterative registration algorithms to overcome the problems of variable lighting and image mis-registration.

- The Gauss-Newton algorithm has been shown to be effective in performing mis-registration correction with large $(\approx 10$ pixel $)$ registration errors.

- Issues relevant to a flaw-detection theory have been presented and applied to test cases in the laboratory. The quantitative tools developed allow an explicit tradeoff between detection probability and the false-error probability. Depending on the flaw model and noise parameters, detection thresholds can be chosen to achieve a given level of performance.

Areas of further work and necessary improvements are identified: 
- Active Inspection Strategies need to be developed to improve data collection upon preliminary detection of a potential flaw. The additional data would be used to improve detection performance and could involve commanding additional sensor platform motions to improve lighting and viewing angles; and selection of different illuminator/camera combinations to get more data.

- The information in the ambient lit image needs to be exploited and used to supplement the image information in the compensated image. In the ideal case, the strobe light should be used only to "probe" or supplement the ambient lit image for additional information.

- Multiple imaging with different electronic shutter settings needs to be investigated in order to improve the dynamic range in both bright and dark regions of image.

- Flaw morphology data needs to be captured by supplementing the imaging sensor with a depth/profile sensor.

- Occlusion data needs to be generated at each vista point to allow the anticipation of previously occluded portions of the scene being mistaken for flaws. This might require data from an additional camera or from an additional image taken near each vista point.

\section{Integrated Sensor End-Effec- tor}

While visual inspection is the primary means of flaw detection, it is only one of the modes available. There are some anomalies, such as errant temperatures and gas leaks, which are not directly detectable by visual information. Therefore, a compact Integrated Sensor End-Effector (ISEE) has been developed to house not only the cameras and lights, but a suite of other sensors. Figure 2 shows the recently constructed device, where the labeled components are:

A Two intensity feedback controlled halogen lamps.

B Two fast pulse strobes flashes.

C A parallel jaw gripper.

D Two color cameras calibrated for stereo viewing.

E Two infrared triangulation proximity sensors.

F A six DOF force/torque sensor.

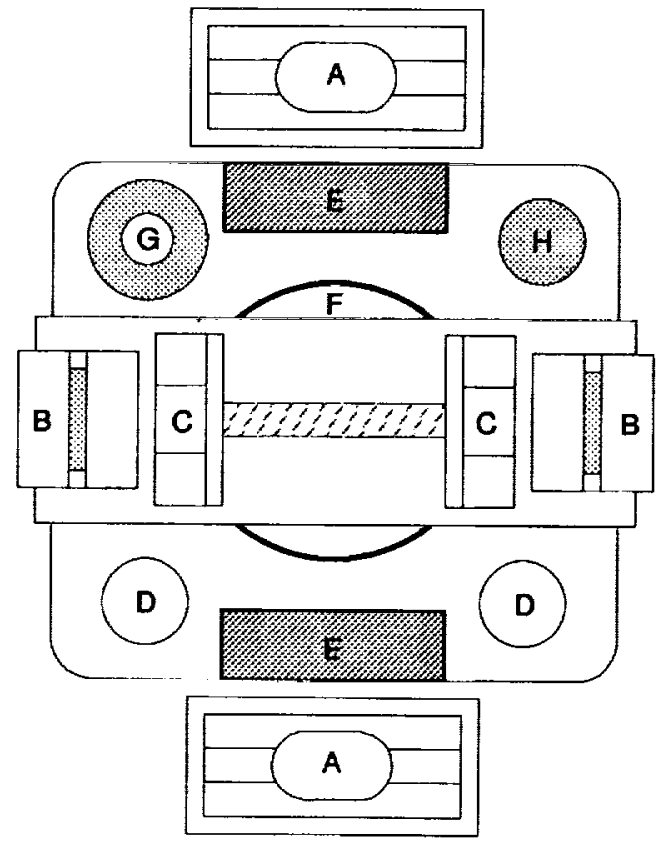

Figure 2: A front view of the ISEE. The lettered components are a described in the text.

G An optical pyrometer with a laser sighting beam.

H A Metal Oxide Semiconductor (MOS) gas/vapor sensor.

Proximity sensing is achieved with two infra-red triangulation sensors, sensitive to approximately $0.75 \mathrm{~m}$. The distance measurements are used for collision avoidance, surface contour following, and surface contour measuring. Temperature sensing is achieved with an infra-red optical pyrometer (8-12 micron wavelength), sensitive to temperatures from 0 to $1000^{\circ} \mathrm{F}$. Gas sensing is achieved with a multi-gas MOS type sensor which changes resistance as a vapor is absorbed. (While it may be possible to employ this gas sensing technology in orbit, we recognize the superiority of using a compact mass spectrometer in the ambient vacuum of space.)

The controlled lights are maintained at a known illumination level by a optical transistor feedback circuit. This makes the illumination independent of current fluctuations and bulb age, and makes precise measurement and camera characterization possible. This lighting is augmented by extremely compact and fast pulse strobes. The strobes provide short duration lighting of intensity on the order of the Sun but only for short, energy saving, single camera frame, bursts. Since the flashes are mounted on the outside surface of the movable parallel jaws of the gripper, the flash illumination angle may be varied as desired.

All components are commercially available, and have been physically and electrically integrated into the com- 


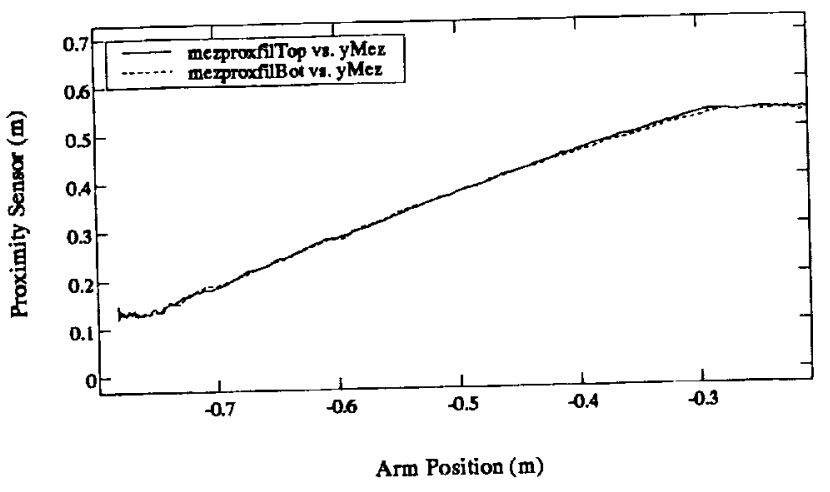

Figure 3: Experimental data showing the filtered proximity measurements from the two proximity sensors as a function of the arm position. The environment surface was at about $-0.81 \mathrm{~m}$.

pact ISEE end-effector, with a resultant mass of approximately $3.5 \mathrm{~kg}$. The force and proximity sensors, as well as the gripper, are not directly used for inspection. Instead, they aid in the control of the robot arm, and therefore, the end-effector. In particular, the proximity sensors can be used for collision prevention and surface tracking. The development of these capabilities is discussed next.

\subsection{Proximity Sensors for Inspection Operations}

A demonstration of the utility of proximity sensor environmental position determination for robot collision avoidance has been performed in a real-time implementation. For these tests, two IDEC/Izumi SA1D triangulating range sensor were used $[11,12]$. Since the sensors have a minimum sensing distance, they were recessed with respect to a parallel jaw gripper which has a length of $11 \mathrm{~cm}$. The sensor values were read through an A/D board by a 68040 processor (VME bus architecture) at a sampling rate of $44 \mathrm{~Hz}$, and the data was digitally low-pass filtered for noise reduction. Figure 3 shows the filtered readings from the two proximity sensors as a function of the robot end-effector position. The response is fairly linear and consistent between the two sensors.

To use the proximity sensor readings for control of the manipulator, the velocity $\dot{x}_{p s}$ in the block diagram of Figure 4 was commanded as a function of the sensed distance. Two different functions were used: collision avoidance and distance servoing. Figure 5 shows these two functions, which are identical except for the dashed segments of the servoing function in regions $D$ and $\mathbf{E}$. The piece-wise continuous formulation was chosen mainly for simplicity in implementation and ease of modification. The value of $V_{j s}$ is the maximum velocity

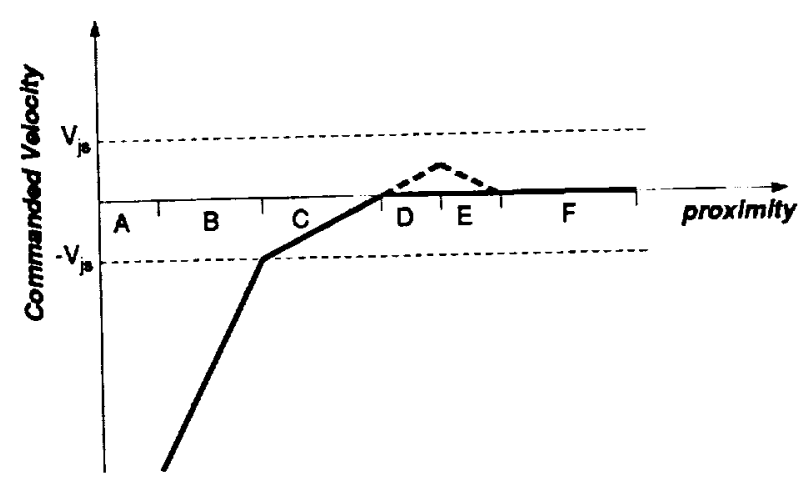

Figure 5: The piece-wise continuous functions of the commanded velocity $\dot{x}_{p s}$ as a function of sensed proximity. The collision avoidance and distance servoing functions are identical except that the latter has positive values indicated by the long dashed line. See the text for a full description.

that can be commanded from the joysticks. Operating region $\mathbf{C}$ provides a collision avoidance velocity command that can not be overridden with a large positive velocity command, $\dot{x}_{j s}$, from the joysticks. Operating region $\mathbf{B}$ allows for quick retreat of the arm if environmental surface protrusions should come into view from the periphery as the the arm is moved tangential to the surface. (It is desirable to restrict the slope and absolute magnitude of the function in this region because of the low sampling rate employed. For instance, had an asymptotic function been employed, there would exist the chance of a very large or rapidly changing velocity command near the asymptote position.) Finally, region $\mathbf{A}$ will typically never be entered since the sensors are recessed, and the sensor is incapable of determining distances at this range.

Regions $\mathbf{D}$ and $\mathbf{E}$ have non-zero values only for distance servoing (the long dash lines in Figure 5). In $\mathbf{D}$, the slope is matched to region $C$, to provide equal acceleration to the servo point between $\mathbf{C}$ and $\mathbf{D}$. The peak value of the distance servoing velocity is restricted, to allow negative joystick commands to overcome it and 'pull' the arm away from the surface. Region $\mathbf{E}$ is provided to make the function continuous. In region $\mathbf{F}$, the sensor can detect distance, but the commanded velocity is zero. Outside of $\mathbf{F}$ the sensor is out of range.

Figures 6 and 7 show the values of $\dot{x}_{p s}$ commanded by the avoidance and servo functions in the real-time implementation. For these measurements $\dot{x}_{p s}$ was not added to $\dot{x}_{r}$, and a simple linear trajectory away from the environmental surface was used for $\dot{x}_{t g}$. 


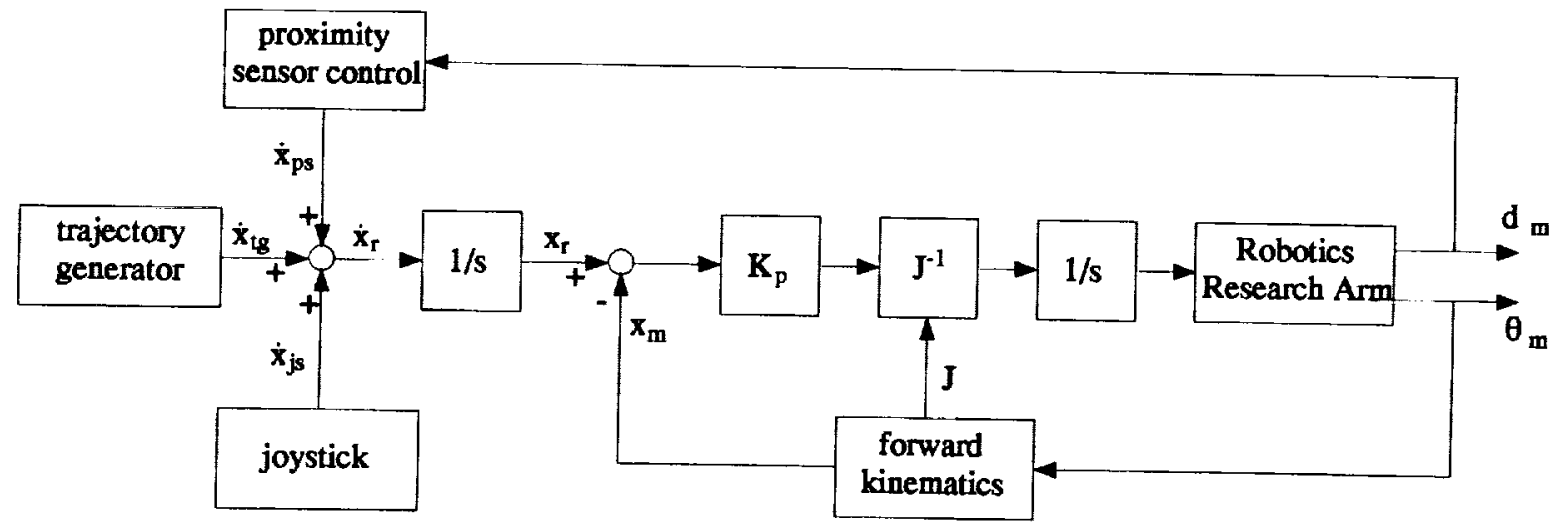

Figure 4: Block diagram of the control system used for the initial tests of proximity sensor collision avoidance.

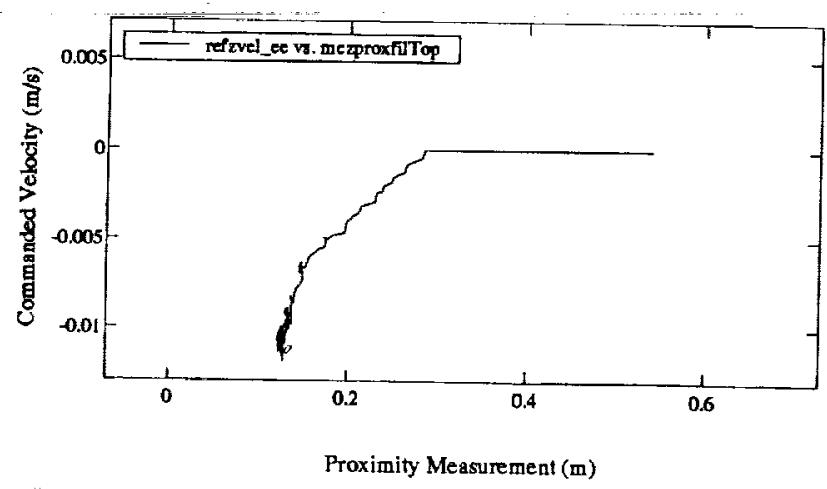

Figure 6: Experimental data showing the commanded repulsion velocity as a function of measured proximity to the environment.

\section{Orbiter and Sunlight Simula- tion}

To demonstrate the capabilities of the inspection system, a one-third scale mock-up of the Space Station Freedom truss has been created. Figure 8 illustrates the mock-up and its components:

A Electrical Orbital Replacement Unit (ORU) which opens to the left on a hinge to reveal electrical connectors and a Cold Plate.

\section{B Tank ORU.}

C Solar Panel.

D Tank and Tubing ORU.

E Simulated hot and cold spots.

F Simulated micrometeor impacts and gas leaks.

The simulated hot and cold spots on the electrical ORU are created using Peltier effect heat pump modules

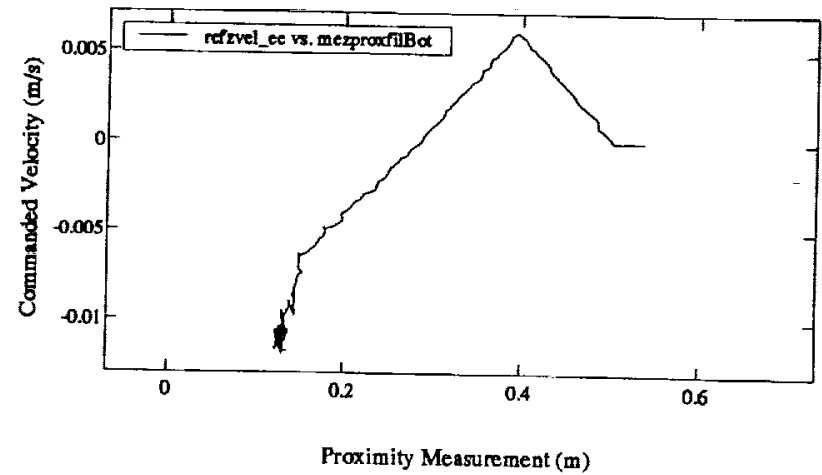

Figure 7: Experimental data showing the commanded distance servo velocity as a function of measured proximity to the environment.

mounted on the inside of the aluminum surface. Since the aluminum has a low emissivity, the outside surface is covered with a circle of Black Kapton to enable the surface temperature to be correctly measured by the optical pyrometer. In the future, the surface temperature may be measured directly by touching it with a thermocouple, eliminating the need for the Kapton.

To introduce a degree of randomness into the inspection process, only two of the Peltier modules are turned on at any time, and the selected direction of electrical current determines if the surface becomes hot or cold. A similar selection is available from amongst the three possible gas "leaks". Each leak uses compressed air to spray a fine mist of household ammonia (to simulate hydrazine) from a small hole on the Tank and Tubing ORU.

Also, random defects may be introduced into the truss mockup through three simple methods. First, screws throughout the truss can be randomly removed to indicate structural defects. Second, small pieces of black tape on pen markings can be placed throughout to simulate micrometeorite impact sites. Third, entire 


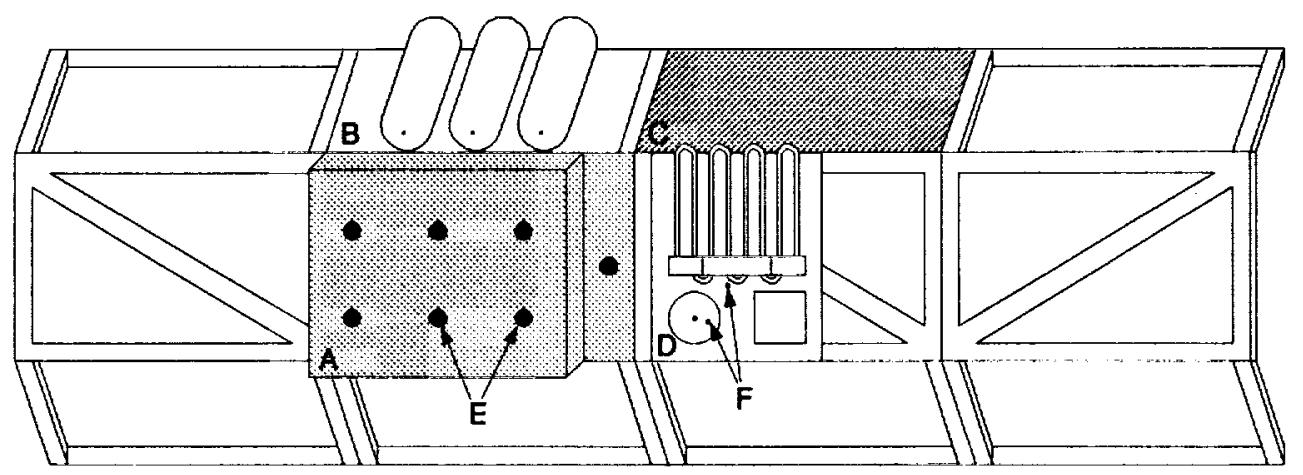

Figure 8: The mock-up of the SSF truss. The lettered components are described in the text.

components, such as tanks, can be replaced with defective versions.

Finally, to simulate the space environment around the truss, the mock-up and the inspection robot have been placed in a room darkened by black curtains [4]. The operator can view the mockup and inspection operations from one of three stereo camera views or from a window of the SSF cupola mock-up, in which the inspection station is situated. Simulated sunlight is provided by the Solar Illumination Simulator, discussed next.

\subsection{Robotic Lighting Control for Solar Illumination Simulation}

Traditional solar simulators are designed for thermal tests of actual spacecraft [5]. To accomplish this, they utilize large vacuum chambers to house the spacecraft, and collimated lighting from arrays of xenon arc lamps. Brightness up to an order of magnitude greater than solar intensity is possible. To test the effects of changing lighting direction, the entire spacecraft is rotated while the illumination remains constant. While this approach is necessary for pre-flight spacecraft testing, it is simply not practical for robotic system prototype development.

Alternatively, we have developed a small scale simulator which effectively mimics the relative motion of the Sun in the sky, while still providing realistically scaled illumination levels [13]. Figure 9 is a photograph of the simulator, a 1500 Watt arc lamp mounted on a four degrees-of-freedom, computer controlled platform. Its ability to pan/tilt/translate, as well as modify the beam shape, enable the illumination angle of the scene to be varied at rates equal to those experienced in low Earth orbit, and maintain a constant illumination flux just as the Sun provides. While the simulated solar illumination is only $1.5 \%$ that of true orbital sunlight, Section 2.1 has previously described the compensating adjustments of controlled lighting position, strobe light-

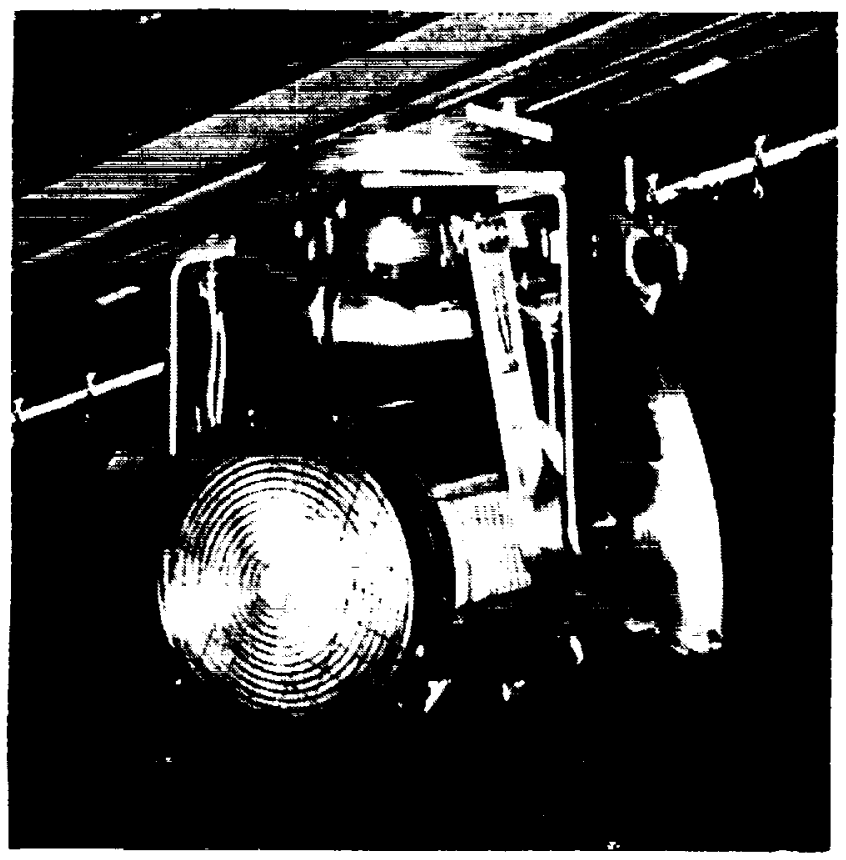

Figure 9: A photograph of the solar illumination simulation system's robotic hardware.

ing pulses, and camera exposure times, provided by the inspection system [1]. Therefore, the lighting conditions are a realistic test for machine inspection algorithms and human operators.

Figure 10 shows the solar illumination simulator as a five DOF system, which is represented by its state vector of configuration variables, $\boldsymbol{\theta}=(\rho, \theta, \varphi, \lambda, \gamma)$, where:
$\rho, \theta, \varphi$ Spherical coordinates from the lamp cen- ter to the projected spot center.
$\lambda$ Travel of lamp from its origin frame.
$\gamma \quad$ Lamp focus parameter indicating position of bulb carriage on internal lead screw.

These parameters have the following ranges: 


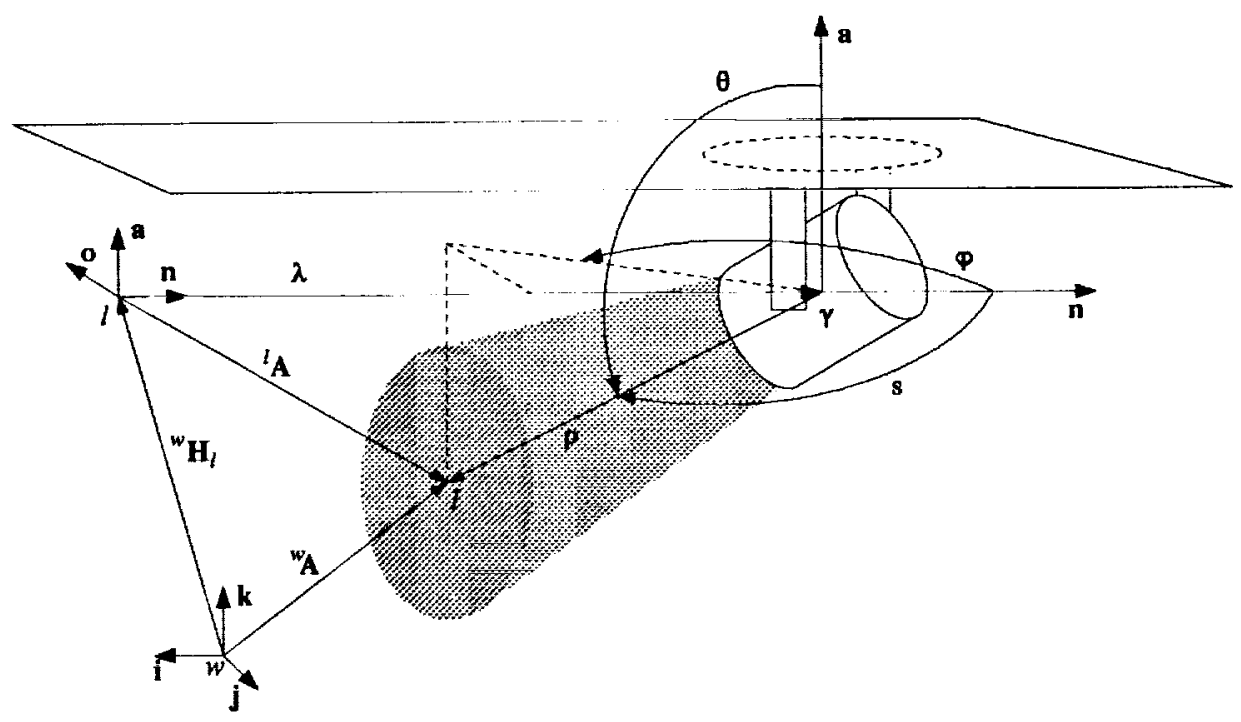

Figure 10: The configuration and task coordinates for the solar illumination simulator system.

\begin{tabular}{|c|c|c|}
\hline & MIN & MAX \\
\hline$\rho$ & $0.15 \mathrm{~m}$ & $\infty$ \\
$\theta$ & $90^{\circ}$ & $180^{\circ}$ \\
$\varphi$ & $60^{\circ}$ & $120^{\circ}$ \\
$\lambda$ & 0 & $4.5 \mathrm{~m}$ \\
$\gamma$ & 0 & $0.076 \mathrm{~m}$ \\
\hline
\end{tabular}

The corresponding task state vector, $\mathbf{x}=\left({ }^{w} \mathbf{A}, s, \mathcal{I}\right)$, is composed of the following variables which are also shown in Figure 10:
${ }^{w}$ A Cartesian vector from world frame to cen- ter of projected spot.
$s \quad$ Beam angle from the lamp frame $\mathbf{n}$ axis.
$\mathcal{I}$ Light intensity at the center of the spot on the environment.

The task vector is obtained from the configuration vector through the forward kinematics: $\mathbf{x}=\mathbf{F}(\boldsymbol{\theta})$.

Finally, it is important to note that although the kinematics has five DOF, only four are actuated. In the configuration space, the unactuated and unmeasured DOF is the radial distance from the lamp to the surface, $\rho$. It's value is calculated from the user specified world coordinates, ${ }^{w} \mathbf{A}$. The controller is open-loop for this variable since no real-time measurement of $\rho$ is possible.

In the task space, the unactuated and unmeasured DOF is the light intensity at the surface. Maintenance of the intensity is performed open-loop based on the calculated value of $\rho$ and an optics model which has been experimentally verified [13].

\section{Conclusions}

This paper has presented the details of some of the technology developed for telerobotic inspection of space platforms such as SSF. Primary amongst the inspection technologies has been visual inspection using computer processing of images from robotically controlled cameras. The processing provides ambient light compensation, registration correction, and automatic flaw detection based on the described flaw models. Secondary inspection and other sensory data are provided by gas, temperature, proximity, and force sensors integrated into the compact ISEE end-effector. This device has been described and the proximity sensor based control of collision avoidance and surface following has been highlighted. Finally, a complete description has been given for the simulated orbiter defects and the space environment lighting. This simulation environment has allowed more rigorous testing of the developed inspection devices and methods.

\section{Acknowledgments}

The research described in this paper was carried out by the Jet Propulsion Laboratory, California Institute of Technology, under a contract with the National Aeronautics and Space Administration. Reference herein to any specific commercial product, process, or service by trade name, trademark, manufacturer, or otherwise, does not constitute or imply its endorsement by the United States Government or the Jet Propulsion Laboratory, California Institute of Technology. 


\section{References}

[1] J. Balaram. Automated Visual Change Detection For Remote Surface Inspection. Internal Engineering Memorandum 3474-93-004, Jet Propulsion Laboratory, California Institute of Technology, Pasadena, CA, September 1993.

[2] F. Fisher and C Price. Space Station Freedom External Maintenance Task Team - Final Report. Technical report, Johnson Space Center, Houston, Texas, July 1990.

[3] P. Gill, W. Murray, and M. Wright. Practical Optimization. Academic Press.

[4] S. Hayati et al. Remote Surface Inspection System. Journal of Robotics and Autonomous Systems, 11(1):45-59, 1993.

[5] Our Captive Space: JPL Space Simulator Facilities. Brochure 400-68, Jet Propulsion Laboratory, California Institute of Technology, Pasadena, California, September 1993.

[6] W. S. Kim. Graphical Operator Interface for Space Telerobotics. In IEEE International Conference on Robotics and Automation, pages 761-768, Atlanta, Georgia, May 2-6 1993.

[7] L. Murr and Kinard W. Effects of Low Earth Orbit. American Scientist, 81-2:152-165, 1993.

[8] T. See et al. Meteoroid and Debris Impact Features Documented on the Long Duration Exposure Facility. Technical Report 24608, Johnson Space Center, Houston, Texas, August 1990.

[9] H. Seraji, M. Long, and T. Lee. Motion Control of 7-DOF Arms: The Configuration Control Approach. IEEE Transactions on Robotics and Automation, 9(2), April 1993.

[10] H. Van Trees. Detection, Estimation, and Modulation Theory: Part 1. John Wiley.

[11] R. Volpe. Year End Task Report of The Prototype Safety System for Robots Near Flight Hardware. Internal Engineering Memorandum 3474-93-006, Jet Propulsion Laboratory, Pasadena, CA, October 1993.

[12] R. Volpe. A Survey and Experimental Evaluation of Proximity Sensors for Space Robotics. In submission to The IEEE International Conference on Robotics and Automation, May 1994.

[13] R. Volpe and D. McAffee. A Robotic Lighting System for Solar Hlumination Simulation. In submission to The IEEE International Conference on Robotics and Automation, May 1994. 